This is a pre-copy-editing, author produced PDF of an article accepted for publication in Medicine \& Science in Sporta \& Exercise following peer review. The definitive publisher-authenticated version - Wilson, John, Haddow, Olivia, Clark, Johnathon, Irwin, Laura, Easton, Chris, Fudge, Barry, Kayser, Bengt and Pitsiladis, Yannis (2006) Tri-axial accelerometry as a simple means to estimate fast running speeds, Medicine \& Science in Sports \& Exercise, 2008, 38 (Supplement 5), S.501.is available online at http://journals.lww.com/acsm-msse/ Fulltext/2006/05001/Tri_axial_accelerometry_as_a_simple_means_to.2969.aspx 


\section{Tri-axial Accelerometry as a Simple Means to Estimate Fast Running Speeds}

John Wilson ${ }^{1}$, Olivia Haddow ${ }^{1}$, Jonathon Clark ${ }^{1}$, Laura Irwin ${ }^{1}$, Chris Easton ${ }^{1}$, Barry Fudge $^{1}$, Bengt Kayser ${ }^{2}$, Yannis Pitsiladis ${ }^{1} .{ }^{1}$ International Centre for East African Running Science (ICEARS), University of Glasgow, Glasgow, United Kingdom. ${ }^{2}$ University of Geneva, Geneva, Switzerland.

Little is known about the energy cost of running in the field as most research has been conducted in laboratory settings. Assessment of physical activity using accelerometery is becoming more frequent mainly due to the ease of its use and relatively low cost. Previous studies have failed to observe a linear relationship between accelerometer counts and fast running speeds. For example, activity counts leveled off at approximately 10000 counts $\cdot \mathrm{min}^{-1}$ at a running speed of $9 \mathrm{~km} \cdot \mathrm{hr}^{-1}$ when measured using uni-axial accelerometry (Brage et al. Med. Sci. Sports Exerc. 35: 1447-1454, 2003). PURPOSE: To determine the relationship between activity counts measured by tri-axial accelerometry and 1) running speed, 2) heart rate (HR), and 3) oxygen uptake $\left(\mathrm{VO}_{2}\right)$. METHODS: Eight trained subjects completed a maximal discontinuous incremental running test on a motorized treadmill (Woodway PPS55 Med, Weil am Rhein, Germany) at speeds corresponding to 8, 10, 12, 14, 16, 18, and $20 \mathrm{~km} \cdot \mathrm{hr}^{-1}$, or until volitional exhaustion. Subjects completed 3 min of exercise at each running speed, followed by 3-5 min recovery. Activity counts (tri-axial accelerometer, $3 \mathrm{dNX}{ }^{\mathrm{TM}}$, BioTel Ltd, Bristol, UK), HR (Suunto t6, Suunto Oy, Vantaa, Finland), and gas exchange variables (breath-by-breath using a quadrupole mass spectrometer, QP9000, Morgan Medical, Gillingham, Kent, UK) were measured throughout exercise. RESULTS: Activity counts increased linearly with treadmill running speeds $(r=0.985, p<0.001)$ (Figure 1$)$. In addition, activity counts during running was significantly correlated with $\mathrm{HR}(r=0.988, p<0.001)$ and $\mathrm{VO}_{2}(r=$ $0.990, p<0.001)$. CONCLUSIONS: The finding of a linear relationship between activity counts measured by tri-axial accelerometry and running speeds up to worldclass marathon running pace raises the intriguing possibility that this technology could be used for metabolic monitoring of endurance athletes during free-living, training and competition when combined with other easily obtained measures (e.g. HR and foot-ground contact times).

Supported by BioTel Ltd, Bristol, UK.

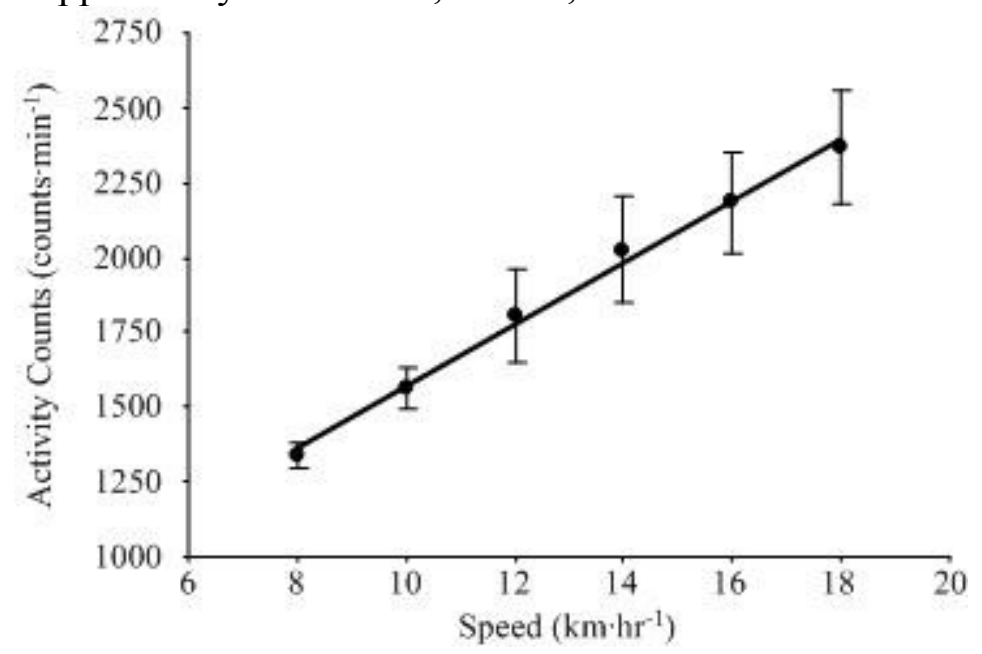

